Cinémas

Revue d'études cinématographiques

Journal of Film Studies

\title{
Une nouvelle direction à la revue : l'avenir et le passé d'une discipline en développement
}

\section{André Gaudreault}

Volume 26, numéro 2-3, printemps 2016

URI : https://id.erudit.org/iderudit/1039363ar

DOI : https://doi.org/10.7202/1039363ar

Aller au sommaire du numéro

Éditeur(s)

Cinémas

ISSN

1181-6945 (imprimé)

1705-6500 (numérique)

Découvrir la revue

Citer ce document

Gaudreault, A. (2016). Une nouvelle direction à la revue : l'avenir et le passé

d’une discipline en développement. Cinémas, 26(2-3), 7-15.

https://doi.org/10.7202/1039363ar d'utilisation que vous pouvez consulter en ligne. 


\section{Une nouvelle direction à la revue: l'avenir et le passé d'une discipline en développement}

Le signataire du présent texte a eu l'insigne honneur et l'immense privilège de diriger la revue Cinémas durant dix-sept années (à partir du volume 10, en 1999). À compter du présent numéro, c'est Richard Bégin, professeur au Département d'histoire de l'art et d'études cinématographiques de l'Université de Montréal, qui est désormais au gouvernail. Pour assurer son avenir, la revue pourra compter sur ce chercheur, jeune mais déjà confirmé, et dont la feuille de route est à tous égards remarquable: on lui doit notamment d'avoir ouvert un nouveau champ de recherche - la mobilographie, cette "écriture de la mobilité» rendue possible par les divers dispositifs portables que la "révolution" numérique a mis à notre disposition —, qui se développe à une vitesse stupéfiante et qui attire de nombreux candidats à la maîtrise et au doctorat.

Passer ainsi à la direction de Cinémas ne tient pas du saut périlleux pour Richard Bégin, puisqu'il a été le directeur adjoint de la revue à partir de juillet 2015 et qu'il est membre de son comité de direction depuis cinq ans, ce qui lui a permis de se familiariser avec l'ensemble des opérations. Le nouveau directeur a donc de l'expérience dans la gestion d'une revue, sans compter qu'il en a déjà lui-même fondé une — la revue en ligne Écranosphère - il y a quelques années.

Je suis donc à même d'en témoigner: Richard sait faire, mais il sait aussi parfaire. Heureusement, car ses responsabilités sont énormes: en ces temps difficiles où le monde de l'édition est en bouleversement continuel, il devra veiller au devenir d'une revue qui peut s'enorgueillir de la réussite qui a marqué l'histoire de ses vingt-six années d'existence, depuis sa fondation par ce duo de visionnaires qu'étaient Michel Larouche et Denise Pérusse (alors respectivement professeur et chercheuse à l'Université de Montréal), en ces temps «reculés» (le début 
des années 1990) où le champ des études cinématographiques était encore, au Canada du moins, dans un état fort embryonnaire.

Il en fallait de la vision, me semble-t-il, pour avoir l'audace de penser qu'une revue savante, comme on dit, pouvait être viable au Québec, dans une discipline qui devait encore faire ses preuves dans l'institution universitaire avant de pouvoir s'y faire reconnaitre pleinement. Au Québec, en 1990, les docteurs en cinéma se comptaient sur les doigts d'une main... Il n'y avait alors aucun programme de maitrise - et encore moins de doctorat - en cinéma, ni au Québec, ni dans le reste du Canada.

On peut imaginer l'angoisse du directeur d'une revue d'études cinématographiques devant un tel désert: qui donc va écrire des articles dignes d'être publiés dans ma revue? En effet, une revue savante, la chose est connue, se doit de publier des textes savants, qui doivent, par définition, être écrits par des... savants. Or, en ces temps-là, des "savants" en cinéma, il n'y en avait, au Québec en tout cas, qu'un tout petit contingent. Certes, une revue peut compter sur des chercheurs étrangers comme auteurs, mais elle doit aussi pouvoir s'appuyer sur les forces locales, ne serait-ce que pour arriver à réunir dans un même lieu les membres de son comité de direction (la visioconférence restait un procédé assez lourd).

Quand on y pense, fonder au Québec une revue du type de Cinémas en 1990, dans l'état peu avancé où se trouvait alors le champ des études cinématographiques, c'était une manière indirecte de lancer un appel pour que ce champ se développe dans l'immédiat. Et comme c'était dans l'air du temps, c'est exactement ce qui arriva : à l'Université de Montréal, certes, mais aussi dans l'ensemble du système universitaire québécois, de même que dans celui du reste du Canada; ainsi, aussi, que de par le vaste monde.

La discipline des études cinématographiques a en effet connu, ces trente dernières années, une croissance exponentielle. Au Québec, les principales universités ont rapidement développé de nouveaux programmes en cinéma, au premier cycle, certes, mais aussi à la maitrise. La création, en 2007, du tout premier 
programme de doctorat en études cinématographiques au Canada (ici même, à l'Université de Montréal) est venue couronner le tout, d'autant qu'elle a bientôt été suivie par l'instauration de programmes doctoraux à l'Université Concordia (Film and Moving Image Studies), à l'Université York (Cinema and Media Studies), à l'Université Wilfrid-Laurier (English and Film Studies) et à l'Université de Toronto (Cinema Studies). Ce sont là quelques-uns des indices récents de l'accession du champ des études cinématographiques au rang de discipline reconnue. À son niveau, la revue Cinémas a joué un rôle dans cet accès de la discipline au "saint des saints" de l'institution universitaire, en participant notamment à sa légitimation et au resserrement des normes scientifiques y ayant cours, et en offrant aux jeunes chercheurs une plate-forme pour publier leurs articles ou encore pour se rompre à la direction de numéros thématiques.

Dans des témoignages sollicités en 2007 par Cinémas, deux chercheurs de niveau international soulignaient déjà l'importance de la revue pour la discipline. Laurent Jullier (Sorbonne Nouvelle), par exemple, écrivait :

Avec la disparition d'Iris, on peut affirmer que Cinémas est la dernière revue à perpétuer une tradition des approches multidisciplinaires et théoriques de haut niveau en matière de cinéma, et donc une chance pour tous les universitaires désireux de faire avancer la recherche en cinéma.

Dans la même veine et à la même occasion, Rick Altman (Université de l'Jowa) affirmait quant à lui :

A multilingual treasure, Cinémas is the only film journal constructing intellectual bridges between Canada, Europe and the U.S. Regularly breaking new multidisciplinary ground, Cinémas has become one of the most important resources for students and scholars of cinema everywhere.

Effectivement, en raison de son ouverture aux articles en anglais (chaque numéro comprend au moins un article dans cette langue, quand ce n'est pas deux, voire trois), la revue Cinémas est devenue un point de rencontre entre les traditions universitaires anglophone et francophone, à la confluence des continents américain et européen. 
Soutenue financièrement depuis plus de deux décennies par le Conseil de recherches en sciences humaines du Canada et par le Fonds de recherche du Québec - Société et culture, la revue a, depuis sa création, son siège au Département d'histoire de l'art et d'études cinématographiques de l'Université de Montréal. Elle est l'une des réalisations ayant marqué et stimulé le développement de la Section cinéma et jeu vidéo dudit département — développement qu'on peut qualifier de fulgurant si on se rappelle qu'à la naissance de Cinémas, en 1990, la section en question ne comptait que trois postes (et demi) de professeurs réguliers, alors qu'elle en compte maintenant quatorze, et n'administrait que deux programmes d'enseignement (tous au premier cycle), comparativement à sept (aux trois cycles) à l'heure actuelle. La revue doit son succès et sa longévité à la constante collaboration des membres de la section, ainsi qu'à celle du département dans son ensemble, qui a fourni le soutien essentiel à son développement et à sa survie, sur le plan tant financier que logistique.

La préparation du présent numéro, qui sera le premier à paraître sous la nouvelle direction, se fait d'ailleurs au moment où le département célèbre ses cinquante années d'existence. Le fait que la première revue d'études cinématographiques francophone à voir le jour au Canada soit née à l'Université de Montréal, et dans ce département en particulier, ne relève pas du hasard, comme le montrent les découvertes que nous avons faites récemment dans les archives de l'université, en préparant justement ce cinquantième anniversaire ${ }^{1}$. Nous venons en effet de découvrir que c'est dès sa naissance, en 1965, que le département a inclus dans ses objets d'étude le cinéma - lequel fait donc partie, pour ainsi dire, de son ADN - et que c'est l'Université de Montréal qui a été, dès l'année suivante, la première université canadienne à offrir un programme en études cinématographiques: pas que des cours de cinéma, mais, on me permettra d'insister, un programme; ce qui n'a pas la même signification puisque, dans le cas de la création d'un programme, ce ne sont pas les instances «locales» (c'est-à-dire départementales) qu'il faut convaincre du bien-fondé de la chose, mais bel et bien les instances supérieures de l'administration de l'université, ce qui représente un 
défi d'une autre dimension pour un sujet, le cinéma, qui était souvent vu à l'époque plus comme un simple divertissement que comme une forme d'art (ainsi qu'en témoigne le document reproduit à la figure 1 , qui commence par cette phrase

\section{Au dèpartement d'fistoire de l'art}

\section{Cerpifical d'épudes cinématographiques}

Le cinĕma a maintenant droit de cité à l'Université. Son caractère artistique et son importance sociologique sont reconnus par tous. Les 350 ciné-clubs qui existent dans la Province de Quêbec prouvent que le cinéma Est plus qu'ure simple entreprise commerciale de spectacles et que le film est devenu l'un des plus importants moyens d'expression artistique.

L'Universitē a mis sur pied un progıamme d'études permettant aux Étudiants de la Faculté des lettres d'obtenir un certificat d'études supérieures intitulé: "Études cinématographiques", certificat valable soit pour l'obtention d'une licence libre ès lettires, soit pour celle d'une licence avec mention histoire de l'art .

Ce nouveau progranme a êté établi par le directeur du département d'histoire de l'art, le professeur L.V. RANDALL. I! permettra la formation de professeurs en cinématographie et de critiques cinématographiques.

Ce nouvel enseignement répond à l'un des voeux du rapport Parent, à savoir que: à partir de 1970, l'éducation cinématogra. phique ne soit confiée qu'à des enseignants qualifiěs dans cette spécialité.

\section{Programme des cours}

Les cours seront connés par des spécialistes du cinerma. Le programme détaillé sera publiě ultérieurement. II traitera aussi bien de l'histoire du cinémia, de l'esthétique, que de la réalisation d'un film et de la portée sociologique du cinéma.

Fig. 1. Notice (non signée) publiée dans Hebdo-Information, le journal de l'Université de Montréal, le 9 mai 1966 (p. 4). Source: Division de la gestion de documents et des archives de l'Université de Montréal. 
rien moins qu'anodine: "Le cinéma a maintenant droit de cité à l'Université ").

C'est dire non seulement que l'Université de Montréal a été, en 2007, la première université canadienne à offrir un programme de doctorat en cinéma, mais aussi que cette même université croyait déjà assez au cinéma, dès 1966, pour donner son aval à la création d'un programme devant "permettr[e] la formation de professeurs en cinématographie et de critiques cinématographiques", et pour recruter à l'international un candidat de haute valeur pour venir y enseigner et y développer ledit programme; ce qui explique l'engagement comme professeur à l'Université de Montréal, de 1966 à 1971, d'un historien et théoricien comme Jean Mitry, alors au faîte de sa "gloire".

Cela intéressera sûrement les lecteurs de la revue d'apprendre que cette ouverture à la res cinematografica de la part d'un département d'histoire de l'art (ce n'est qu'en 2003 que le département changera son nom pour y inclure la mention «études cinématographiques") est vraisemblablement due à l'influence d'un penseur important et imposant comme Erwin Panofsky, dont on a découvert qu'il était un ami proche du "professeur L. V. Randall» dont parle le document reproduit à la figure 1 , celui-là même qui a fondé le département et qui a le mérite d'y avoir inclus ab ovo le cinéma comme objet d'étude.

Randall était un juif allemand ${ }^{2}$ qui, à l'instar de Panofsky, avait dû s'exiler pour fuir le nazisme dans les années 1930. Or, non seulement Panofsky est un éminent historien de l'art, mais il a aussi la particularité d'avoir écrit un texte précurseur sur le cinéma $^{3}$. Dans une lettre du 6 juillet 1966 (un peu moins de deux mois après l'annonce officielle de la création du certificat en études cinématographiques) adressée à Randall (avec qui il entretient une correspondance assez soutenue, dans laquelle l'un et l'autre s'interpellent sur un mode intime et amical: "Dear Ludi » d'un côté, "My dear Pan» de l'autre), Panofsky fait justement référence à l'article qu'il a publié trente ans auparavant, en rappelant qu'il a été l'un des premiers à considérer les films comme des formes artistiques (voir la figure 2): 
That there is a new degree awarded by your University for studies in film-making is of great interest to me. I was one of those who started the whole idea of taking the films as an art form $[\ldots]$ by $[\ldots]$ writing an often reprinted article on "Style and Medium in the Motion Pictures." This was as early as 1934 or so when few respectable people, especially intellectuals, confessed to a liking for the movies $[\ldots]$

\author{
Juls 6, 1966
}

\author{
Professor Lewls Randell \\ Université de Montríal \\ Casa Postale 6128 \\ Montróal, Canada \\ Dear Ind1:
}

Thank you ever so much for your letter of June 30th which reached me on the day before my departure for Europe - and, of course, congratulations both to yourself and to your University on your professorship honoris cansa. I am sure that the University will be well eerved, and some activity is good for the sowl, quite particularly if the body harboring this soul begins to show the inevitable consequences of getting older.

From this point of view - but not from this point of view alone - I should have been delighted to accept your Invitation to Montreal. But, as Erasmus sald: "Quil potest non video." We are not going to return unt11 the end of September, and then I an taken up with a seminar to be given at New York University where I am st11ll the nominal Incumbent of the so-called Samuel F. B. Morse Professorshlp. And after the Chriatmas vacation I have to teach a little at Prinoeton Univeraity. But this does not, of course, dindnish my gratitude for having been thought of.

That there is a new degree awarded by your Unl versity for studies in film-malding is of great interest to me. I was one of those who started the whole Ides of taiding the films as an art form vider willen by participating in the foundation of the FIIn ISbrary at the Karserum of Fodern Art and writing an often reprinted article on "Style and Medium In the Motion Plotures," Th1s was as early as 1934 or so when few respectable people, especially Intelleotuals, confessed to a liking for the movies, and now there is a Full Chalr of CInematology at New York University as woll as a rather ambitious "C1nematologioal Soclety" founded by the incumbent of that chair, a man named Robert Gessner who is a little ambitious but may be able to help with the organization of that new enterprise. However, you probably know all this anyurgy.

With m renewed thanks, and all best wishes in which mo new wife Joins me unbelkannterneloe,

Yours as ever,

BPsre

Frudn Panofakg

Fig. 2. Lettre d'Erwin Panofsky adressée à Lewis Randall, directeur (et fondateur) du Département d'histoire de l'art de l'Université de Montréal (datée du 6 juillet 1966). Source: Fonds Panofsky, Archives of American Art, Washington (merci à Nicolas Herman). 
Sa lettre témoigne par ailleurs du fait que la discipline des études cinématographiques en est encore à ses débuts aux ÉtatsUnis, tout aussi bien :

\section{[...] now there is a Full Chair of Cinematology at New York University as well as a rather ambitious "Cinematological Society" founded by the incumbent of that chair, a man named Robert Gessner who is a little ambitious but may be able to help with the organization of that new enterprise.}

C'est donc dans le contexte favorable et stimulant de ce département dynamique que la revue Cinémas est née - vingt-cinq ans après la correspondance citée entre Randall et Panofsky qu'elle s'y est développée et qu'elle a fini par devenir, en un certain sens, une institution. La revue a publié en un quart de siècle les articles de près de 400 chercheurs et étudiants universitaires des cycles supérieurs, surtout d'Europe et d'Amérique. Elle a également pu compter sur l'expertise de la communauté scientifique pour appliquer le rigoureux système d'évaluation par les pairs qui a été a mis en place dès le début et qui continue d'être scrupuleusement respecté.

Bien entendu, la revue Cinémas doit aussi son succès et sa longévité à la confiance que lui témoignent ses lecteurs et ses abonnés, année après année. Nous leur en savons sincèrement gré.

On me permettra de profiter de cette fin de mandat pour remercier du fond du cœur tous les membres du comité de direction et du comité de rédaction qui ont participé à la vie de la revue pendant la période où je l'ai dirigée, ainsi que les secrétaires de rédaction successives, qui m’ont secondé, et sans le dévouement desquelles je n'aurais pu exercer mes fonctions: Monica Normand, Lisa Pietrocatelli, Marnie Mariscalchi et Charlotte Brady-Savignac. Merci aussi à tous les collaborateurs de la revue, au premier plan desquels nos correcteurs et correctrices, qui par leur zèle constant font de Cinémas une revue qui se corrige!

La revue va maintenant devoir affronter une série de défis, en raison de la numérisation galopante que notre monde connaît, et aussi en raison des tendances actuelles qui favorisent le libre accès, une valeur en laquelle nous croyons, 
Richard et moi, même si la chose reste problématique, notamment sur le plan budgétaire, pour les revues. Je suis sûr que le nouveau directeur, en dépit des difficultés qui ne manqueront pas de se présenter, saura assurer à la revue un avenir à la hauteur de son passé.

\author{
André Gaudreault \\ Directeur de la revue de 1999 à 2016 \\ Membre du Comité de direction depuis 1993
}

\title{
NOTES
}

1. Les informations qui documentent ce que j'avance ici résultent de deux vagues de recherches dans les archives de l'université: la première vague a été le fait, en 20112012, de Kim Décarie et de Stéphanie Croteau, dans le cadre d'une recherche menée sous ma direction pour l'Observatoire du cinéma au Québec; la deuxième vague a été réalisée par Mathieu Boivin, Simon Dor et Olivier Tremblay, en 2015-2016, dans le cadre d'une recherche menée sous la direction de Sarah Guérin et de Nicholas Herman en vue des célébrations du $50^{\circ}$ anniversaire du Département d'histoire de l'art et d'études cinématographiques de l'Université de Montréal.

2. Son véritable nom était Ludwig Rosenthal, qu'il a changé en Ludovic (alias Louis, alias Lewis) Randall; ses amis l'appelaient Ludi. Nicholas Herman, qui est maintenant conservateur au Schoenberg Institute for Manuscript Studies de l'Université de Pennsylvanie, mène depuis quelques années une recherche biographique et historique sur Ludwig Randall et sa collection d'œuvres d'art.

3. Voir Panofsky 1936, 1937 et 1947. Cet article issu d'une conférence donnée en 1934 a été réédité à plusieurs reprises; son titre a d'ailleurs évolué au fil des ans, pour passer de "On Movies» (1936), à "Style and Medium in the Moving Pictures" (1937), puis à "Style and Medium in the Motion Pictures» (1947): on remarquera la gradation qui va du "vulgaire" movies au populaire moving, pour arriver finalement au plus raffiné motion.

\section{RÉFÉRENCES BIBLIOGRAPHIQUES}

Panofsky 1936: Erwin Panofsky, "On Movies", Bulletin of the Department of Art and Archaeology of Princeton University, 1936, p. 5-15.

Panofsky 1937 : Erwin Panofsky, "Style and Medium in the Moving Pictures", Transition, $\mathrm{n}^{\circ}$ 26, 1937, p. 121-133.

Panofsky 1947 : Erwin Panofsky, "Style and Medium in the Motion Pictures", Critique: A Review of Contemporary Art, vol. 1, n 3, 1947, p. 5-28 ("Style et matière du septième art", dans Trois essais sur le style, Paris, Gallimard, 1996, p. 109-145).

Une nouvelle direction à la revue : l'avenir et le passé d'une discipline en développement 